\title{
Representatividade e precisão na estimativa da densidade e área basal na floresta nacional do Tapajós
}

\author{
Tatiana da Cunha CASTRO ${ }^{1 *}$, Ademir Roberto RUSCHEL ${ }^{2}$, João Olegário Pereira de CARVALHO ${ }^{3}$, \\ Edson Marcos Leal Soares RAMOS ${ }^{1}$, Jaqueline Macedo GOMES ${ }^{4}$ \\ ${ }^{1}$ Universidade Federal do Pará, Belém, PA, Brasil \\ ${ }^{2}$ Embrapa Amazônia Oriental, Belém, PA, Brasil \\ ${ }^{3}$ Universidade Federal Rural da Amazônia, Capitão Poço, PA, Brasil. \\ ${ }^{4}$ Universidade Estadual da Região Tocantina do Maranhão, Imperatriz do Maranhão, MA, Brasil. \\ E-mail: ccastro.tatiana@gmail.com
}

Recebido em julho/2018; Aceito em outubro/2018.

RESUMO: O objetivo do estudo foi avaliar a eficiência de parcelas na estimativa da densidade e área basal de uma floresta manejada na Amazônia. A densidade e área basal foram calculadas utilizando dados de um censo florestal realizado em 144 ha e dos dados de medição realizada em 48 parcelas permanentes de 0,25 ha cada (amostra de $12 \mathrm{ha}$ ), instaladas nos 144 ha segundo um processo aleatório simples. Para testar a eficiência amostral na estimativa dessas variáveis foram calculados o erro real relativo, o erro de amostragem e a intensidade amostral para diferentes níveis de inclusão de diâmetro mínimo das árvores no inventário. $\mathrm{O}$ erro real foi calculado para seis níveis de inclusão de DAP, considerando árvores com diâmetro a partir de $25 \mathrm{~cm}$, o erro de amostragem foi calculado para 10 níveis de inclusão de DAP, considerando árvores com diâmetro a partir de $5 \mathrm{~cm}$; e o cálculo da intensidade amostral foi realizado para populações finitas. O limite de erro amostral admissível foi de $10 \%$. A amostragem utilizada na área foi suficiente para gerar resultados com alto nível de precisão, podendo ser aplicada em florestas densas com densidade e área basal semelhantes às da floresta estudada.

Palavras-chave: censo florestal, unidades amostrais, erro amostral, eficiência amostral.

\section{Representativeness and precision in the estimation of density and basal area in the Tapajós national forest}

\begin{abstract}
The objective of the study was to evaluate the efficiency of plots in the estimation of the density and basal area of a managed area in Amazon. The density and basal area were calculated from data of a forest census carried out in 144 ha and data from a measurement carried out in 48 permanent plots of 0.25 ha each (12 ha sample), installed in the same144 ha according to a simple random process. In order to test the sampling efficiency in the estimation of these variables, we calculated the actual relative error, the sampling error and the sample intensity for different inclusion levels of minimum tree diameter in the inventory. The actual error was calculated for six inclusion levels, considering trees with diameter from $25 \mathrm{~cm}$, the sampling error was calculated for 10 inclusion levels, considering trees with diameter from $5 \mathrm{~cm}$, and the calculation of the sampling intensity was performed for finite populations. The admissible sampling error limit was $10 \%$. The sampling used in the area was sufficient to generate results with a high level of precision, being able to be applied in dense forests with density and basal area similar to those of the studied forest.
\end{abstract}

Keywords: forest census, sampling units, sampling error, sample efficiency.

\section{INTRODUÇ̃̃O}

Para utilizar de forma sustentável as florestais naturais é primordial conhecer suas características e sua dinâmica. Uma ferramenta utilizada para essa finalidade é o inventário florestal, que serve de base para a elaboração dos planos de manejo florestal sustentável, por possibilitar a coleta de dados qualitativos e quantitativos da floresta. No entanto, essas informações devem ser precisas e confiáveis, por isso os gestores florestais devem primar por um inventário de qualidade.

Normalmente os recursos disponíveis para o manejo de florestas são limitados, incluindo desde a atividade de inventário florestal, por isso é necessário otimizar as estratégias de amostragem, para reduzir os seus custos, porém mantendo a precisão (MATEO et al., 2018). Os inventários podem ser classificados em relação à obtenção dos dados, em dois tipos: inventário a $100 \%$ de intensidade, também denominado de censo, onde se medem todos os indivíduos da comunidade e se obtêm os valores reais ou parâmetros da comunidade; e inventário amostral, onde são empregadas técnicas de amostragem na comunidade, medindo-se parte da mesma, nesse caso a amostra gera estimativas dos parâmetros da comunidade (SANQUETTA et al., 2014).

Um dos grandes problemas nos inventários florestais na Amazônia é a grande variabilidade de metodologias empregadas, principalmente no que diz respeito ao tamanho $\mathrm{e}$ à forma das unidades amostrais e definição do diâmetro mínimo de inclusão (OLIVEIRA et al., 2014). Por isso, para se obter informações com precisão em um inventário amostral deve-se definir corretamente o processo de amostragem a ser utilizado, o tamanho e a forma das unidades amostrais e a intensidade amostral a ser aplicada na área (UBIALLI et al., 
2009). A metodologia escolhida deve propiciar eficiência com alta precisão e exatidão (KERSHAW JR. et al., 2017; SANQUETTA et al., 2014) com a maior redução de custos possível (MIRANDA et al., 2015). A escolha do método de amostragem depende da finalidade do inventário, das condições locais e das características da população (ARAUJO et al., 2018). Além disso, é importante considerar na escolha da metodologia, que ao se utilizar um processo de amostragem é gerado um erro de amostragem, que é o erro que se comete por medir apenas parte da população, e quanto menor for esse erro mais precisas serão as estimativas obtidas (UBIALLI et al., 2009). Assim, as parcelas amostrais devem proporcionar estimativas dentro de um limite máximo de erro de amostragem admissível, para que os resultados possam ser utilizados para a tomada de decisões do manejo florestal.

$\mathrm{Na}$ presente pesquisa buscou-se responder à questão: as parcelas amostrais de inventário florestal contínuo utilizadas em áreas experimentais na Floresta Nacional do Tapajós (Flona Tapajós) são eficientes para estimar com precisão os parâmetros da comunidade arbórea estudada? Testou-se a hipótese de que as amostras utilizadas na "área experimental Km 114" na Flona Tapajós são adequadas e em número suficiente para gerar com eficiência e precisão informações dendrométricas a serem utilizadas em Planos de Manejo Florestal Sustentável na Amazônia. Assim, o objetivo do presente estudo foi testar a eficiência de 48 parcelas quadradas de 0,25 ha que vêm sendo utilizadas nos inventários contínuos de uma área de pesquisa em manejo florestal localizada na Flona Tapajós. Essa análise foi feita para diferentes categorias de diâmetro mínimo de inclusão de árvores no inventário, por meio do cálculo do erro real relativo, erro de amostragem e da intensidade amostral.

Os resultados indicam a margem de confiabilidade da aplicação da metodologia em diferentes situações, contribuindo assim para aumentar o conhecimento sobre a aplicação de técnicas de amostragem para inventários florestais na Amazônia.

\section{MATERIAL E MÉTODOS}

2.1. Caracterização da área de estudo

A área experimental situa-se na Floresta Nacional do Tapajós, município de Belterra, Pará, à altura do km 114 da BR 163, Rodovia Santarém-Cuiabá, entre as coordenadas geográficas $03^{\circ} 18^{\prime} 31,84364^{\prime \prime}$ - 03 19' 21,48821" de latitude Sul e $54^{\circ} 56^{\prime} 27,96367^{\prime \prime}-54^{\circ} 56^{\prime} 15,13091^{\prime \prime}$ de longitude a Oeste.

O clima da região é tropical chuvoso, caracterizado por ser quente e úmido. A temperatura média anual é de $27^{\circ} \mathrm{C}$, com umidade relativa do ar média de $87 \%$ e precipitação anual acumulada de $1.758 \mathrm{~mm}$, conforme os dados coletados nos últimos cinco anos (Outubro/2012 a Setembro/2017) na estação meteorológica de Belterra (INMET, 2017). Os solos da região são do tipo Latossolo Amarelo distrófico e Argissolo Amarelo distrófico (OLIVEIRA JUNIOR et al. 2015). A vegetação da área de estudo é classificada como Floresta Ombrófila Densa (IBGE, 2012). Em 1982 foi realizada uma exploração florestal planejada na área experimental (144 ha), onde foram colhidas em média 12 árvores $\mathrm{ha}^{-1}$ e um volume médio de $90 \mathrm{~m}^{3} \mathrm{ha}^{-1}$, considerado por Gomes et al. (2018a) como sendo uma colheita de alta intensidade.

\subsection{Coleta de dados}

Os dados utilizados neste estudo foram obtidos a partir de um censo florestal, realizado na área (144 ha) em 2014, considerando árvores com DAP $\geq 25 \mathrm{~cm}$ e a partir dos dados de um inventário realizado em 2012 em 48 parcelas permanentes de $50 \mathrm{~m}$ x $50 \mathrm{~m}$.

As 48 parcelas de $50 \mathrm{~m}$ x $50 \mathrm{~m}$ foram estabelecidas na área segundo um processo aleatório simples, formando uma amostra de 12 ha e uma intensidade amostral de $8,3 \%$. O delineamento foi estabelecido atendendo sugestões para capturar a heterogeneidade entre as unidades de amostra (AVILA et al., 2015). Em cada parcela foram inventariadas as árvores com DAP (diâmetro medido a $1,30 \mathrm{~m}$ do solo) $\geq 5 \mathrm{~cm}$.

\subsection{Análises dos dados}

A eficiência da amostragem foi testada para a densidade (árvores ha $\left.{ }^{-1}\right)$ e área basal $\left(\mathrm{m}^{2} \mathrm{ha}^{-1}\right)$, utilizando os dados obtidos no censo e os dados obtidos nas 48 parcelas.

Após o cálculo da densidade e área basal para os dois inventários (censo e parcelas) foi realizada a comparação usando a diferença entre os valores reais dos parâmetros e os valores estimados pela amostragem. A proporção da diferença entre esses valores dividida pelo valor real obtido no censo possibilitou a determinação do erro real relativo (ER). O erro real relativo foi calculado para seis níveis de inclusão de diâmetro: DAP $\geq 25 \mathrm{~cm}, \mathrm{DAP} \geq 30 \mathrm{~cm}, \mathrm{DAP} \geq 35 \mathrm{~cm}, \mathrm{DAP} \geq$ $40 \mathrm{~cm}, \mathrm{DAP} \geq 45 \mathrm{~cm}$ e DAP $\geq 50 \mathrm{~cm}$. Adicionalmente, para comparar os valores de densidade e de área basal obtidos nas parcelas com os obtidos no censo, aplicou-se o teste t para uma amostra, ao nível de $5 \%$ de significância.

Para a determinação dos erros de amostragem das estimativas da densidade e da área basal foi utilizado o procedimento de cálculo da amostragem aleatória simples. $\mathrm{O}$ erro de amostragem foi calculado a partir dos dados das parcelas, considerando 10 níveis de inclusão de diâmetro: $\mathrm{DAP} \geq 5 \mathrm{~cm}, \mathrm{DAP} \geq 10 \mathrm{~cm}, \mathrm{DAP} \geq 15 \mathrm{~cm}, \mathrm{DAP} \geq 20 \mathrm{~cm}$, $\mathrm{DAP} \geq 25 \mathrm{~cm}, \mathrm{DAP} \geq 30 \mathrm{~cm}, \mathrm{DAP} \geq 35 \mathrm{~cm}, \mathrm{DAP} \geq 40 \mathrm{~cm}$, $\mathrm{DAP} \geq 45 \mathrm{~cm}$ e DAP $\geq 50 \mathrm{~cm}$.

$\mathrm{O}$ erro de amostragem relativo foi calculado conforme a fórmula descrita em Péllico Netto; Brena (1997) (Equação 1).

$$
E_{r}= \pm \frac{t \cdot s_{\overline{\mathrm{X}}}}{\overline{\mathrm{X}}} \cdot 100
$$

em que: $t=$ valor tabelado da distribuição de Student (GL; $\alpha=0,05)$; $S_{\overline{\mathrm{X}}}=$ erro padrão; e $\overline{\mathrm{X}}=$ média.

Para determinar o número ideal de unidades amostrais a serem instaladas na área utilizou-se a fórmula da intensidade amostral para populações finitas de acordo com Péllico Netto e Brena (1997) (Equação 2), considerando um limite de erro de $10 \%$ e probabilidade de $95 \%$.

$$
n=\frac{N \cdot t^{2} \cdot s_{x}^{2}}{N \cdot E^{2}+t^{2} \cdot s_{x}^{2}}
$$

em que: $n=$ número ideal de unidades amostrais; $N=$ número total de unidades amostrais da comunidade; $t=$ valor tabelado da distribuição de Student $(\mathrm{GL} ; \alpha=0,05) ; s_{x}^{2}=$ variância da média da variável analisada; e $E=$ expectância do erro.

Essa análise foi realizada para os 10 níveis de inclusão de DAP considerados no cálculo do erro de amostragem. 


\section{RESULTADOS}

As estimativas do processo de amostragem aleatória simples (AS) foram inferiores aos valores paramétricos observados no censo, tanto para a densidade quanto para a área basal. Apenas quando foram consideradas árvores com DAP $\geq$ $25 \mathrm{~cm}$, o valor estimado para a densidade foi superior ao valor paramétrico (Tabela 1).

Para as parcelas que subestimaram as variáveis analisadas, a subestimação para a densidade variou de $5,38 \%$ a $13,89 \%$, enquanto que para a área basal variou de $7,14 \%$ a $15,71 \%$ (Tabela 1). Como se observa, os erros reais relativos foram maiores para a área basal, em todos os níveis de inclusão de DAP. Mas, apesar das parcelas terem subestimado a densidade e a área basal, os valores estimados foram próximos dos valores paramétricos obtidos no censo (Figura 1). O resultado do teste $t$ mostra que a diferença entre esses valores não é significativa para os níveis de inclusão de DAP $\geq 25 \mathrm{~cm}, \geq 30$ $\mathrm{cm}, \geq 35 \mathrm{~cm}$ e $\geq 50 \mathrm{~cm}$ para a densidade e para o nível de inclusão de DAP $\geq 25 \mathrm{~cm}$ para a área basal, indicando que a amostragem é suficiente para estimar a densidade e área basal da população quando se considera esses níveis de inclusão de DAP.

Tabela 1. Densidade e área basal registradas na amostragem aleatória simples (AS) (valor estimado) e no censo (valor real), com os respectivos erros reais relativos (ER\%) e valores do teste $t$ nos diferentes níveis de inclusão de DAP.

Table 1. Density and basal area measured in the simple random sampling (estimated value) and in the forest census (actual value) with the respective relatives actual errors (ER\%) and t-test values in the different DBH inclusion levels.

\begin{tabular}{ccccc|cccc}
\hline \multirow{2}{*}{ Nível de inclusão de DAP } & \multicolumn{4}{c}{ Densidade $\left(\right.$ árv ha $\left.{ }^{-1}\right)$} & \multicolumn{4}{c}{ Área basal $\left(\mathrm{m}^{2}\right.$ ha $\left.^{-1}\right)$} \\
\cline { 2 - 9 } & AS & Censo & ER $(\%)$ & Teste t & AS & Censo & ER $(\%)$ & Teste t \\
\hline Árv com DAP $\geq 25$ & 126,75 & 122,92 & $-3,12$ & $0,84^{\text {ns }}$ & 18,16 & 19,56 & 7,14 & $1,59^{\text {ns }}$ \\
Árv com DAP $\geq 30$ & 87,00 & 91,94 & 5,38 & $1,33^{\text {ns }}$ & 15,83 & 17,73 & 10,74 & $2,19^{*}$ \\
Árv com DAP $\geq 35$ & 61,42 & 66,5 & 7,64 & $1,61^{\text {ns }}$ & 13,72 & 15,66 & 12,36 & $2,28^{*}$ \\
Árv com DAP $\geq 40$ & 42,92 & 49,32 & 12,98 & $2,33^{*}$ & 11,71 & 13,78 & 15,01 & $2,48^{*}$ \\
Árv com DAP $\geq 45$ & 31,50 & 36,58 & 13,89 & $2,27^{*}$ & 10,12 & 12,00 & 15,71 & $2,44^{*}$ \\
Árv com DAP $\geq 50$ & 23,83 & 27,15 & 12,24 & $1,93^{\text {ns }}$ & 8,85 & 10,35 & 14,43 & $2,10^{*}$ \\
\hline
\end{tabular}

ns: não significativo (Teste t $\mathrm{p}>0,05$ ); ${ }^{*}$ significativo (Teste $\mathrm{t} p<0,05$ ).
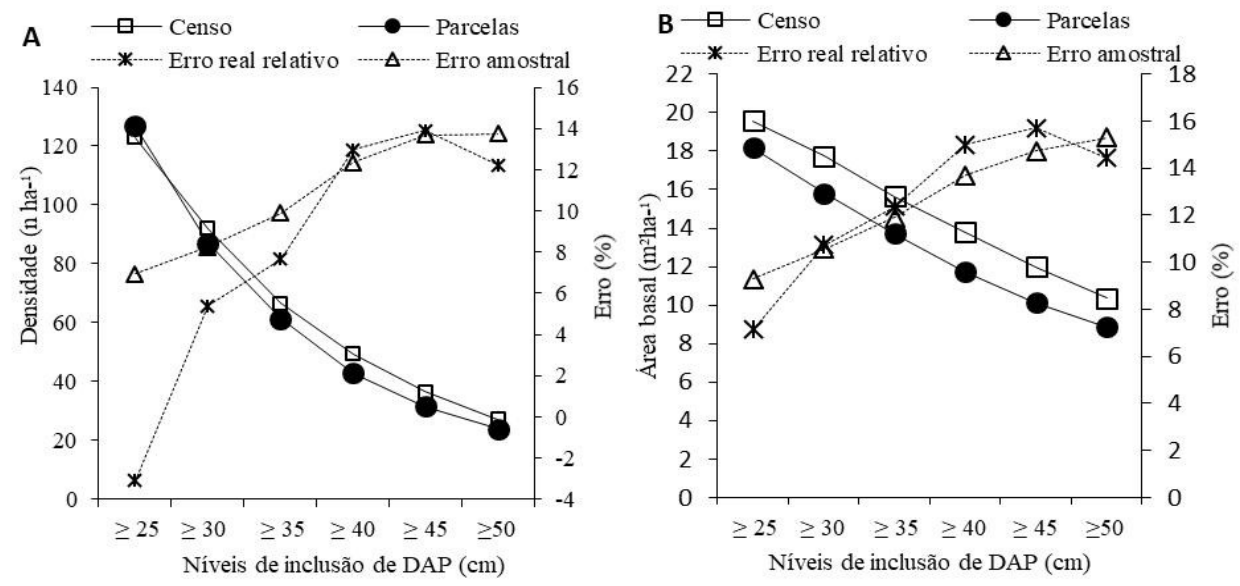

Figura 1. A) Número de árvores por hectare e B) área basal $\left(\mathrm{m}^{2} \mathrm{ha}^{-1}\right)$ no censo de 2014 e nas parcelas (medição 2012), com os respectivos erros reais relativos e erros amostrais por nível de inclusão de DAP.

Figure 1. A) Number of trees per hectare and B) basal area $\left(\mathrm{m}^{2} \mathrm{ha}^{-1}\right)$ in the $100 \%$ intensity inventory (2014) and in the plots (2012), with the respective relative actual errors and sampling errors per inclusion DBH levels.

Considerando os erros amostrais estimados para a densidade e área basal, observa-se que ficaram abaixo do limite de erro requerido, que é de $10 \%$, até quando se considerou árvores com DAP $\geq 25 \mathrm{~cm}$ (Figura 2), o que significa que a amostragem utilizada na área do experimento foi suficiente para estimar com precisão as duas variáveis em estudo até esse nível de inclusão de DAP. Entretanto, nos níveis de inclusão de DAP $\geq 40 \mathrm{~cm}, \geq 45 \mathrm{~cm} \mathrm{e} \geq 50 \mathrm{~cm}$, as estimativas dos erros amostrais para a densidade ficaram acima de $10 \%$. E para a área basal o erro foi superior a $10 \%$ nos níveis de inclusão de DAP $\geq 30 \mathrm{~cm}$; DAP $\geq 35 \mathrm{~cm}$; DAP $\geq 40 \mathrm{~cm}, \geq 45 \mathrm{~cm} \mathrm{e} \geq 50 \mathrm{~cm}$. É importante considerar que nos inventários amostrais ou diagnósticos aceitam-se erros em torno de $20 \%$ para árvores com DAP $\geq 50 \mathrm{~cm}$ de espécies comerciais. Assim, os erros amostrais estimados para a densidade $(13,8 \%)$ e área basal $(15,3 \%)$ seriam aceitáveis.
Essa tendência de aumento dos erros amostrais com o aumento do diâmetro mínimo de inclusão pode ser observada claramente na Figura 2. Comportamento similar foi observado para o erro real relativo mostrado na Figura 1.

Os erros amostrais foram maiores para a área basal, em todos os níveis de inclusão de DAP, assim como se observou para o erro real relativo. À medida em que se aumenta o diâmetro mínimo de inclusão do inventário, mais unidades amostrais precisam ser instaladas na área para atingir o limite de erro requerido no inventário $(10 \%)$, o que pode ser confirmado quando se observa a Figura 3.

Para estimar com precisão a densidade e a área basal das árvores com DAP $\geq 5 \mathrm{~cm}$, seria suficiente uma amostra $81 \%$ menor para a densidade e $65 \%$ menor para a área basal do que a utilizada na área, considerando esse nível de inclusão de DAP. Entretanto, para o último nível de inclusão de DAP $\geq 50$ 
$\mathrm{cm}$, onde foram observados os maiores erros amostrais (Figura 2), seria necessária uma amostra duas vezes maior (99 parcelas) para estimar com precisão a área basal das árvores com DAP $\geq 50 \mathrm{~cm}$ (Figura 3). Por ouro lado, no inventário diagnóstico o erro amostral requerido exigido para espécies comerciais com DAP $\geq 50$ (diâmetro mínimo de corte estabelecido pela legislação brasileira) é de $20 \%$. Nessa situação, o número de unidades amostrais usadas na área poderia ser suficiente para estimar com precisão a densidade e a área basal.

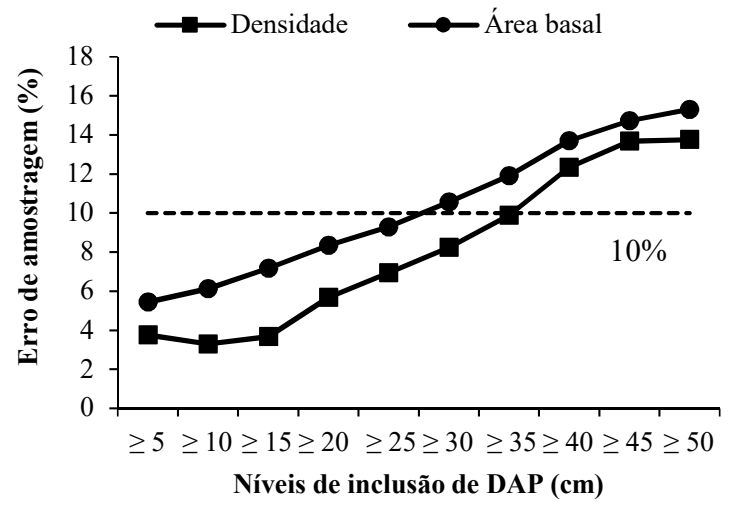

Figura 2. Estimativa do erro amostral por nível de inclusão de DAP, para a densidade e área basal, em uma área de 144 ha (amostra de 12 ha $-8,3 \%$ de intensidade amostral) na Floresta Nacional do Tapajós, Belterra, Pará.

Figure 2. Estimate of the sampling error by DBH inclusion level for density and basal area in a 144 ha area (12 ha sample; $8.3 \%$ sampling intensity) in the Tapajos National Forest, Belterra, Para.

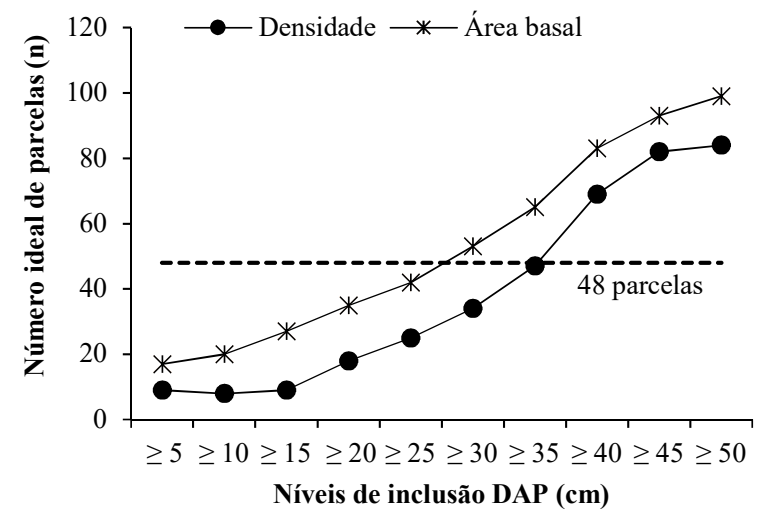

Figura 3. Número ideal de parcelas de $50 \mathrm{~m} \times 50 \mathrm{~m}$ a serem instaladas por nível de inclusão de DAP, considerando o limite de erro amostral de $10 \%$, para a densidade e área basal, em uma área de 144 ha (amostra de 12 ha $-8,3 \%$ de intensidade amostral) na Floresta Nacional do Tapajós, Belterra, Pará.

Figure 3. Optimal number of $50 \mathrm{~m}$ x $50 \mathrm{~m}$ plots to be established by DBH inclusion level, considering a $10 \%$ sampling limit error, for density and basal area, in a 144 ha area (12 ha sample; 8,3\% sampling intensity) in the Tapajos National Forest, Belterra, Para.

\section{DISCUSSÃO}

A tendência do erro real relativo para a estimativa da densidade e da área basal foi diretamente proporcional ao aumento do diâmetro mínimo de inclusão, ou seja, os maiores erros foram observados nos maiores níveis de inclusão de DAP.

A estimativa da eficiência amostral para a estrutura da floresta, utilizando a comparação dos valores paramétricos obtidos no censo florestal com os valores estimados por meio da amostragem com parcelas, também foi avaliada em outros estudos como, por exemplo, de Augustynczik et al. (2013) e de Cavalcanti et al. (2011). Na avaliação da estrutura das populações de determinadas espécies, devem ser empregadas dimensões mais especificas de parcelas (VIANA et al., 2018), assim como métodos diferentes de amostragem, atentando para as limitações de cada espécie (BOWERINGA et al., 2018) e para o tamanho e idade da floresta, além das condições do ambiente local (LOMBARDI et al., 2015). Há casos em que o erro associado à mensuração pode influenciar na estimativa, por exemplo, do volume da madeira (GOMES et al, 2018b).

Cavalcanti et al. (2011) compararam os valores de abundância, área basal e volume de árvores com DAP $\geq 40 \mathrm{~cm}$, estimados em 70 parcelas de $50 \mathrm{~m}$ x $400 \mathrm{~m}$, com os valores registrados no censo. Nesse estudo os valores estimados para a densidade e área basal também foram bem próximos dos valores paramétricos encontrados no censo. Os resultados desse estudo, assim como os resultados da presente pesquisa, indicam que para reduzir o erro para as árvores de maiores diâmetros é necessário aumentar a intensidade amostral.

A tendência de aumento dos erros reais e amostrais com o aumento do diâmetro mínimo de inclusão está diretamente relacionada com a distribuição diamétrica das árvores na floresta, que é na forma de "J invertido", distribuição comum em florestas naturais tropicais (GONÇALVES; SANTOS, 2008; REIS et al., 2010). Esse tipo de distribuição é caracterizado pela menor concentração de árvores nas maiores classes de diâmetro, o que justifica os maiores erros encontrados para os maiores níveis de inclusão de DAP. Desta forma, aumentar o número ou o tamanho das parcelas poderiam ser estratégias adotadas para reduzir os erros dos inventários de árvores com diâmetros superiores.

No presente estudo, a área basal mostrou ser mais exigente do que a densidade, apresentando estimativas menos precisas. Isso ocorre por que a área basal depende diretamente do diâmetro, uma variável que tem alta variabilidade em florestas naturais. Assim, os maiores erros são gerados na estimativa da área basal em comparação com a densidade, que não depende diretamente do diâmetro. Conforme Nascimento et al. (2004), quanto mais heterogênea e irregular for a floresta, maior deverá ser o número de unidades amostrais para estimar a área basal.

\section{CONCLUSÕES}

A eficiência amostral variou de acordo com os níveis de inclusão de DAP das árvores. Os maiores erros, tanto o real quanto o de amostragem, foram observados para as populações de árvores de maiores diâmetros. Portanto, o número de amostras deve ser aumentado se houver interesse em estimar estoque e produção apenas de árvores com diâmetros maiores que $40 \mathrm{~cm}$ em parcelas de $50 \mathrm{~m}$ x $50 \mathrm{~m}$.

Considerando um erro amostral de $10 \%$, o número de parcelas utilizadas na área foi suficiente para estimar, com precisão, a densidade da população de árvores, com exceção daquelas com DAP maior que $40 \mathrm{~cm}$. Foi suficiente também para estimar a área basal das árvores, com exceção daquelas com DAP maior que $30 \mathrm{~cm}$. Portanto, o número e tamanho de parcelas estabelecidas na área é suficiente para gerar resultados com alto nível de precisão, podendo ser aplicada em florestas densas de terra firme com densidade e área basal semelhantes à densidade e área basal da floresta estudada. 
6. REFERENCIAS

ARAUJO, E. J. G.; PELLICO NETTO, S.; MORAIS, V. A.; DAVID, H. C.; CURTO, R. A.; SCOLFORO, J. R. S. Métodos de amostragem de área variável para a regeneração natural de Eremanthus erythropappus (DC.) MacLeish. Floresta, Curitiba, v.48, n.2, p.265-276, 2018. DOI: http://dx.doi.org/10.5380/rf.v48i2.55726

AUGUSTYNCZIK, A. L. D; MACHADO, S. A.; FIGUEIREDO FILHO, A; PELLICO NETTO, S. Avaliação do tamanho de parcelas e de intensidade de amostragem em inventários florestais. Scientia Forestalis, Piracicaba, v. 41, n. 99, p. 361-368, 2013.

AVILA, A. L.; RUSCHEL, A. R.; CARVALHO, J. O. P.; MAZZEI, L.; SILVA, J. N. M.; LOPES, J. C.; ARAUJO, M. M.; DORMANN, C. F.; BAUHUS, J. Medium-term dynamics of tree species composition in response to silvicultural intervention intensities in a tropical rain forest, Biological Conservation, Essex, v. 191, p. $577-$ 586, 2015.2 DOI: https://dx.doi.org/10.1016/j.biocon.2015.08.004

BOWERINGA, R.; WIGLEA, R.; PADGETTA, T.; ADAMS, B.; COTEC, D.; WIERSMAA, Y. F. Searching for rare species: A comparison of Floristic Habitat Sampling and Adaptive Cluster Sampling for detecting and estimating abundance. Forest Ecology and Management, Amsterdam, v.407, p. 1-8, 2018. DOI: https://dx.doi.org/10.1016/j.foreco.2017.10.016

CAVALCANTI, F. J. B., MACHADO, S. A; HOSOKAWA, R. T, CUNHA, U. S. Comparação dos valores estimados por amostragem na caracterização da estrutura de uma área de floresta na Amazônia com as informações registradas no censo florestal. Revista Árvore, Viçosa, v. 35, n. 5, p. 1061-1068, 2011. DOI: http://dx.doi.org/10.1590/S010067622011000600012

GOMES, J. M.; CARVALHO, J. O. P.; RUSCHEL, A. R.; SILVA, J. N. M.; RAMOS, E. M. L. S.; CASTRO, T. C.; TOHOMPSON, I. S.; FREITAS, L. J. M. Estoque de duas espécies arbóreas ameaçadas de extinção aos 30 anos após a exploração na Amazônia oriental. Scientia Forestalis, Piracicaba, v. 46, n. 117, p. 41-52, mar. 2018a. DOI: http://dx.doi.org/10.18671/scifor.v46n117.04

GOMES, K. M. A.; RIBEIRO, R. B. S.; GAMA, J. R. V.; ANDRADE, D. F. C. Eficiência na estimativa volumétrica de madeira na Floresta Nacional do Tapajós. Nativa, Sinop, v. 6, n. 2, p. 170-176, mar./abr. 2018b. DOI: http://dx.doi.org/10.31413/nativa.v6i2.5237

GONÇALVES, F. G.; SANTOS, J. R. Composição florística e estrutura de uma unidade de Manejo florestal sustentável na Floresta Nacional do Tapajós, Pará. Acta Amazônica, Manaus, v. 38, n. 2, p. 229-244, 2008. DOI: http://dx.doi.org/10.1590/S0044-59672008000200006

IBGE_Instituto Brasileiro de Geografia e Estatística. Manual técnico da vegetação brasileira. Rio de Janeiro: DEDIT/CDDI; 2012. $271 \mathrm{p}$.

INMET_Instituto Nacional de Meteorologia. Ministério da Agricultura, Pecuária e Abastecimento. Dados Climáticos. Disponível em: $<$ http://www.inmet.gov.br/portal/index.php?r=bdmep $>$. Acesso em 15 out 2017.

KERSHAW JR., J. A.; DUCEY, M. J.; BEERS, T. W.; HUSCH, B. Forest Mensuration. 5. ed. Hoboken: WileyBlackwell - John Wiley \& Sons, 2017. 160 p.
LOMBARDI, F.; MARCHETTI, M.; CORONA, P.; MERLINI, P.; CHIRICI, G.; TOGNETTI, R.; BURRASCANO, S.; ALIVERNINI, A.; PULETTI, N. Quantifying the effect of sampling plot size on the estimation of structural indicators in old-growth forest stands. Forest Ecology and Management, Amsterdam, v. 346, p. 89-97, 2015. DOI: https://dx.doi.org/10.1016/j.foreco.2015.02.011

MATEO, R. G.; GASTON, A.; AROCA-FERNANDEZ, M. J.; SAURA, S.; GARCIA-VIÑAS, J. I. Optimization of forest sampling strategies for woody plant species distribution modelling at the landscape scale. Forest Ecology and Management, Amsterdam, v. 410, p. 104113, 2018 . DOI: https://dx.doi.org/10.1016/j.foreco.2017.12.046

MIRANDA, D. L. C.; FRANCIO, J.; SANTOS, J. P.; SANQUETTA, C. R.; CORTE, A. P. D. Precisão e eficiência relativa de métodos de amostragem em teca. Pesquisa Florestal Brasileira, Colombo, v. 35, n. 83, p.2 47-254, 2015.

DOI: https://dx.doi.org/10.4336/2015.pfb.35.83.638

NASCIMENTO, A. R. T.; SCARIOT, A.; SILVA, J. A.; SEVILHA, A. C. Estimativas de área basal e uso do relascópio de Bitterlich em amostragem de Floresta Estacional Decidual. Ciência florestal, Santa Maria, v. 14, n. 2, p. 169-176, 2004. DOI: http://dx.doi.org/10.5902/198050981816

OLIVEIRA JUNIOR, R. C.; KELLER, M. M.; RAMOS, J. F. F.; BELDINI, T. P.; CRILL, P. M.; CAMARGO, P. B.; VAN HAREN, J. Chemical analysis of rainfall and throughfall in the Tapajós National Forest, Belterra, Pará, Brazil. Ambiente \& Agua, Taubaté, v. 10, p. 263-285, 2015. DOI: http://dx.doi.org/10.4136/ambi-agua. 1552

OLIVEIRA, M. M; HIGUCHI, N.; CELES, C. H.; HIGUCHI, F. G. Tamanho e formas de parcelas para inventários florestais de espécies arbóreas na Amazônia Central. Ciência Florestal, Santa Maria, v.24, n.3, p.645-653, 2014. DOI: http://dx.doi.org/10.5902/1980509815744

PÉLLICO NETTO, S.; BRENA, D. A. Inventário florestal. Curitiba: Editorado pelos autores, 1997. 316 p.

REIS, L. P.; RUSCHEL, A. R; COELHO, A. A.; LUZ, A. S.; MARTINS-DA-SILVA, R. C. V. Avaliação do potencial madeireiro na Floresta Nacional do Tapajós, após 28 anos da exploração florestal. Pesquisa Florestal Brasileira, v. 30 , n. 64, p. 265-281, 2010. DOI: http://dx.doi.org/10.4336/2010.pfb.30.64.265

SANQUETTA, C. R.; DALLA CORTE, A. P.; RODRIGUES, A. L.; WATZLAWICK, L. F. Inventários florestais: planejamento e execução. 3. ed. Curitiba: Multi-graphic Gráfica e Editora, 2014. 406 p. v. 1.

UBIALLI, J. A; FIGUEIREDO FILHO A; MACHADO, S. A.; ARCE, J. E. Comparação de métodos e processos de amostragem para estudos fitossociológicos em uma floresta ecotonal na região norte matogrossense. Floresta, Curitiba, v. 39, n. 3, p. 511-523, 2009. DOI: http://dx.doi.org/10.5380/rf.v39i3.15351

VIANA, A. B. T.; TORRES, C. M. M. E.; SILVA, L. F.; ROCHA, S. J. S. S. Influência do tamanho de parcela no cálculo da distribuição espacial de Anadenanthera peregrina (L.) Speng. e Apuleia leiocarpa J. F. Macbr em uma floresta estacional semidecidual. Nativa, Sinop, v. 6, n. 2 , p. 183-190, mar./abr. 2018. DOI: http://dx.doi.org/10.31413/nativa.v6i2.4938 